Pure and Applied Mathematics Quarterly

Volume 8, Number 4

$1029-1046,2012$

\title{
Simply Connected Fast Escaping Fatou Components
}

\author{
D. J. Sixsmith
}

\begin{abstract}
We give an example of a transcendental entire function with a simply connected fast escaping Fatou component, but with no multiply connected Fatou components. We also give a new criterion for points to be in the fast escaping set.
\end{abstract}

Keywords: transcendental entire functions; wandering domain; fast escaping set.

\section{INTRODUCTION}

Suppose that $f: \mathbb{C} \rightarrow \mathbb{C}$ is a transcendental entire function. The Fatou set $F(f)$ is defined as the set of points $z \in \mathbb{C}$ such that $\left(f^{n}\right)_{n \in \mathbb{N}}$ is a normal family in a neighborhood of $z$. The Julia set $J(f)$ is the complement of $F(f)$. An introduction to the properties of these sets was given in [3].

The fast escaping set $A(f)$ was introduced in [5]. We use the definition $A(f)=\left\{z:\right.$ there exists $\ell \in \mathbb{N}$ such that $\left|f^{n+\ell}(z)\right| \geq M^{n}(R, f)$, for $\left.n \in \mathbb{N}\right\}$, given in [10]. Here, the maximum modulus function $M(r, f)=\max _{|z|=r}|f(z)|$, for $r>0, M^{n}(r, f)$ denotes repeated iteration of $M(r, f)$ with respect to $r$, and $R>0$ can be taken to be any value such that $M(r, f)>r$, for $r \geq R$. We write $M(r)$ when it is clear from the context which function is being considered.

Suppose that $U=U_{0}$ is a component of $F(f)$. If $U \cap A(f) \neq \emptyset$, then $\bar{U} \subset A(f)$ [10, Theorem 1.2]. We call a Fatou component in $A(f)$ fast escaping. Denote by $U_{n}$ the component of $F(f)$ containing $f^{n}(U)$. We say that a component is 
wandering if $U_{n}=U_{m}$ implies that $n=m$. All fast escaping Fatou components are wandering; [5, Lemma 4] and [10, Corollary 4.2].

For a transcendental entire function, all multiply connected Fatou components are fast escaping; [8, Theorem 2] and [10, Theorem 4.4]. The first example of a transcendental entire function with a multiply connected Fatou component was constructed by Baker in [1]. Other examples are found in, for example, [2], [4], [6] and [7].

The only known example of a simply connected fast escaping Fatou component was given by Bergweiler [4], using a quasi-conformal surgery technique from [7]. This function also has multiply connected Fatou components. In fact, in [4], the properties of the multiply connected Fatou components are used to show that the simply connected Fatou components are fast escaping.

This prompts the question of whether a transcendental entire function can have simply connected fast escaping Fatou components without having multiply connected Fatou components. We answer this in the affirmative, using a direct construction and a recent result on the size of multiply connected Fatou components [6, Theorem 1.2] to prove the following.

Theorem 1. There is a transcendental entire function with a simply connected fast escaping Fatou component, and no multiply connected Fatou components.

To prove Theorem 1 we require a new sufficient condition for points to be in $A(f)$, which may be of independent interest.

Theorem 2. Suppose that $f$ is a transcendental entire function. Suppose also that, for $R_{0}>0, \epsilon:\left[R_{0}, \infty\right) \rightarrow(0,1)$ is a nonincreasing function, such that

$$
\epsilon\left(M^{n}(r)\right) \geq \epsilon(r)^{n+1}, \quad \text { for } r \geq R_{0} \text { and } n \in \mathbb{N} .
$$

Define $\eta(r)=\epsilon(r) M(r)$, for $r \geq R_{0}$. Then there exists $R_{1} \geq R_{0}$ such that

$$
A(f)=\left\{z: \text { there exists } \ell \in \mathbb{N} \text { such that }\left|f^{n+\ell}(z)\right| \geq \eta^{n}\left(R^{\prime}\right), \text { for } n \in \mathbb{N}\right\},
$$

for $R^{\prime} \geq R_{1}$.

Note that this is a generalisation of [10, Theorem 2.7], which is obtained from Theorem 2 when $\epsilon$ is constant. 


\section{The Definition of THE FUnCTION}

In this section we define a transcendental entire function, $f$, which has all the properties defined in Theorem 1 . Since $f$ is very complicated, we first outline informally the construction of $f$, starting with simpler functions which only have some of these properties. We then give the full construction. A detailed proof of Theorem 1 is given in subsequent sections.

Consider first a transcendental entire function defined by a power series;

$$
g(z)=z \prod_{k=1}^{\infty}\left(1+\frac{z}{a_{k}}\right)^{2}, \quad 0<a_{1}<a_{2}<\cdots .
$$

The sequence $\left(a_{n}\right)_{n \in \mathbb{N}}$ can be chosen so that the following holds: we can define another sequence, $\left(b_{n}\right)_{n \in \mathbb{N}}$, such that $b_{n}$ is approximately equal to $a_{n},-b_{n}$ is close to a critical point of $g$, and $g\left(-b_{n}\right)$ is close to $-b_{n+1}$. It can then be shown that a small disc centred at $-b_{n}$ is mapped by $g$ into a small disc centred at $-b_{n+1}$. By Montel's theorem, these discs must be in the Fatou set of $g$. Moreover, these discs cannot be in multiply connected Fatou components of $g$ since, by [6, Theorem 1.2], any open set contained in a multiply connected Fatou component of $g$ must, after a finite number of iterations of $g$, cover an annulus surrounding the origin. Finally, it can be shown, by comparing $\left|g\left(-b_{n}\right)\right|$ to $M\left(b_{n}, g\right)=g\left(b_{n}\right)$, that these discs are contained in fast escaping Fatou components of $g$.

However, $g$ does not have all the properties defined in Theorem 1. In particular, by considering the behaviour of $g$ in large annuli which omit the zeros of $g$, it can be shown that $g$ has multiply connected Fatou components. Thus $g$ has very similar properties to the example in [4].

We note that no zero of $g$ can be in a multiply connected Fatou component, since 0 is a fixed point. In order to prevent the existence of multiply connected Fatou components, we add further zeros to the function, along the negative real axis. This requires some care. The addition of too many zeros - for example, spaced linearly along the negative real axis - leads to a breakdown of other parts of the construction. The addition of a zero with modulus insufficiently distant from $a_{n}$ leads to a similar breakdown.

We use [6, Theorem 1.2] to show that only a relatively small number of additional zeros are required. In particular, suppose that $h$ is a transcendental entire function with $h(0)=0$ and with zeros of modulus $r_{0}<r_{1}<r_{2}<\cdots$. 
Then, by [6, Theorem 1.2], $h$ has no multiply connected Fatou components if $\lim _{k \rightarrow \infty} \log r_{k+1} / \log r_{k}$ exists and is equal to 1 .

To use this result, we need to understand the behaviour of $\log a_{n+1} / \log a_{n}$, for large $n$. From the recursive definition that we use to ensure that the sequences $\left(a_{n}\right)_{n \in \mathbb{N}}$ and $\left(b_{n}\right)_{n \in \mathbb{N}}$ have the required properties, (see (3.15)), we find that, for large $n, \log a_{n+1} / \log a_{n}$ is close to $n^{3}$. See (3.9) for a precise statement of how the term $n^{3}$ arises here.

This suggests the following. Define $\mu_{n}=n^{3 / n}$, for $n \in \mathbb{N}$. To simplify some displays we set $\mu_{n, m}=\mu_{n}^{m}$, and observe that $\mu_{n, 0}=1$ and $\mu_{n, n}=n^{3}$, for $n \in \mathbb{N}$. We now define a more complicated transcendental entire function;

$$
h(z)=z \prod_{k=1}^{\infty} \prod_{l=0}^{k-1}\left(1+\frac{z}{a_{k}^{\mu_{k, l}}}\right)^{2}, \quad 0<a_{1}<a_{2}<\cdots
$$

The sequence $\left(a_{n}\right)_{n \in \mathbb{N}}$ in this definition is not the same as in the definition of $g$, but serves the same purpose, and is chosen similarly. This function has zeros of modulus

$$
\cdots, a_{n}, a_{n}^{\mu_{n}}, a_{n}^{\mu_{n, 2}}, \cdots, a_{n}^{\mu_{n, n-1}}, a_{n+1}, a_{n+1}^{\mu_{n+1}} \cdots .
$$

Since it is readily seen that $\mu_{n} \rightarrow 1$ as $n \rightarrow \infty$, this function does not have multiply connected Fatou components. However, two further adjustments are required. Firstly, the zero of modulus $a_{n}^{\mu_{n, n-1}}$ is sufficiently close to the zero of modulus $a_{n+1}$ that the original construction breaks down. We resolve this by omitting this zero. Secondly, the value of $\log a_{n+1} / \log a_{n}$ is not close enough to $n^{3}$, for large $n$, to ensure that $\lim _{k \rightarrow \infty} \log a_{k+1} / \log a_{k}^{\mu_{k, k-2}}=1$. We resolve this by adding one additional zero, which serves no other purpose in the construction. This zero is defined using two additional sequences, $\left(\alpha_{n}\right)_{n \in \mathbb{N}}$ and $\left(\beta_{n}\right)_{n \in \mathbb{N}}$, which we choose to keep $\log a_{n+1} / \log a_{n}$ sufficiently close to $n^{3}$.

Now we are able to indicate the form of the function $f$ in Theorem 1 . Let $f$ be the transcendental entire function;

$$
f(z)=z \prod_{k=3}^{\infty}\left\{\left(1+\frac{z}{a_{k}^{\beta_{k}}}\right)^{2 \alpha_{k}} \prod_{l=0}^{k-2}\left(1+\frac{z}{a_{k}^{\mu_{k, l}}}\right)^{2}\right\},
$$

where $0<a_{3}<a_{4}<\cdots, \alpha_{n} \in\{0,1,2, \ldots\}, \beta_{n} \in \mathbb{R}$, for $n \in \mathbb{N}$. Again, the sequence $\left(a_{n}\right)_{n \in \mathbb{N}}$ in this definition is not the same as that in the definition of $g$ or $h$, but serves the same purpose, and is chosen similarly. The related sequence 
$\left(b_{n}\right)_{n \in \mathbb{N}}$, discussed after the definition of $g$, is defined for $f$ by (3.14). The sequences $\left(\alpha_{n}\right)_{n \in \mathbb{N}}$ and $\left(\beta_{n}\right)_{n \in \mathbb{N}}$ are the two sequences mentioned at the end of the previous paragraph.

The structure of the proof of Theorem 1 is as follows. First, in Section 3, we give the definition of the various sequences in (2.1), and we prove a number of estimates on the modulus of the zeros of $f$. Next, in Section 4, we show that there are no multiply connected Fatou components of $f$. In Section 5 we show that there are intervals on the negative real axis each contained in some Fatou component of $f$. Finally, in Section 6 we prove Theorem 2 and then use this to show that these Fatou components of $f$ are fast escaping. It is clear that Theorem 1 follows from these results.

Remark: Rippon and Stallard asked [10, Question 1] if there can be unbounded fast escaping Fatou components of a transcendental entire function. It can be shown that the Fatou components of the function $f$ are all bounded. Indeed, it is straightforward to prove that the number of zeros of $f$ in the disc $\{z:|z|<r\}$ is $O(\log r)$, and hence that $\log M(r, f)=O\left((\log r)^{2}\right)$. It follows that $f$ has no unbounded Fatou components [10, Theorem 1.9(b)], and, moreover, that the set $A(f)$ has a structure known as a spider's web. For more details we refer to [10].

\section{Defining the SEquences}

In this section we first define the sequences $\left(\alpha_{n}\right)_{n \in \mathbb{N}}$ and $\left(\beta_{n}\right)_{n \in \mathbb{N}}$, and then define the sequences $\left(a_{n}\right)_{n \in \mathbb{N}}$ and $\left(b_{n}\right)_{n \in \mathbb{N}}$.

Recall from Section 2 that $\mu_{n}=n^{3 / n}$ and $\mu_{n, m}=\mu_{n}^{m}$; we also define, for $n \geq 3$,

$$
\sigma_{n}=\sum_{l=1}^{n-2} \mu_{n, l}=\mu_{n} \frac{\mu_{n, n-2}-1}{\mu_{n}-1} .
$$

We define $\left(\alpha_{n}\right)_{n \in \mathbb{N}}$ to be a sequence of integers and $\left(\beta_{n}\right)_{n \in \mathbb{N}}$ to be a sequence of real numbers. Assume that $N_{0}$ is even and chosen sufficiently large for subsequent 
estimates to hold. Set

$$
2 \alpha_{n}= \begin{cases}0, & \text { for } n<N_{0}, \\ N_{0}^{3}+2 N_{0}^{2}+6 N_{0}+2, & \text { for } n=N_{0}, \\ 3 n^{2}+n+6, & \text { for } n>N_{0}, n \text { even } \\ 3 n^{2}+n+4, & \text { for } n>N_{0}, n \text { odd }\end{cases}
$$

Note that $\alpha_{n}$ is an integer, for $n \in \mathbb{N}$. Set

$$
\beta_{n}= \begin{cases}0, & \text { for } n<N_{0}, \\ \frac{1}{\alpha_{n}}\left(n^{4}-\sigma_{n}\right), & \text { for } n \geq N_{0}, n \text { even, } \\ \frac{1}{\alpha_{n}}\left(\frac{n^{3}(2 n-1)}{2}-\sigma_{n}\right), & \text { for } n \geq N_{0}, n \text { odd. }\end{cases}
$$

We observe that these choices imply that

$$
\tau_{n}=\frac{2}{n^{3}}\left(\alpha_{n} \beta_{n}+\sigma_{n}\right)
$$

satisfies

$$
\tau_{n}= \begin{cases}2 n, & \text { for } n \geq N_{0}, n \text { even } \\ 2 n-1, & \text { for } n \geq N_{0}, n \text { odd }\end{cases}
$$

and

$$
2 \alpha_{n}=3 n^{2}+n+3+\tau_{n}-\tau_{n-1}, \quad \text { for } n>N_{0} .
$$

We also define a sequence of integers $\left(T_{n}\right)_{n \in \mathbb{N}}$ by

$$
T_{n}= \begin{cases}n^{3}+2 n-3, & \text { for } n \text { even } \\ n^{3}+2 n-2, & \text { for } n \text { odd. }\end{cases}
$$

Next we prove a result which gives various relationships between these sequences.

Lemma 3.1. The following all hold for the choice of sequences above:

$$
\begin{gathered}
\alpha_{n} \sim \frac{3}{2} n^{2}, \quad \beta_{n} \sim \frac{2}{3} n^{2}, \quad \text { as } n \rightarrow \infty, \\
2 \sum_{k=3}^{n} \alpha_{k}=n^{3}+2 n^{2}+4 n+2+\tau_{n}, \quad \text { for } n \geq N_{0}, \\
1+2 \sum_{k=3}^{n-1} \alpha_{k}+\sum_{k=3}^{n-1} \sum_{l=0}^{k-2} 2=n^{3}+\tau_{n-1}=T_{n}, \quad \text { for } n \geq N_{0},
\end{gathered}
$$


and

$$
\mu_{n, 2}<\beta_{n}<\mu_{n, n-3}, \quad \text { for large } n
$$

Proof. The first half of (3.7) is immediate from (3.2). Now, by (3.2), (3.3) and (3.4),

$$
\beta_{n} \sim \frac{2 n}{3}\left(n-\frac{\sigma_{n}}{n^{3}}\right), \quad \text { as } n \rightarrow \infty
$$

We have that

$$
\frac{x}{2} \leq \log (1+x) \leq x, \quad \text { for } 0<x<\frac{1}{2}
$$

Putting $x=\mu_{n}-1$, we obtain

$$
\frac{3}{n} \log n \leq \mu_{n}-1 \leq \frac{6}{n} \log n, \quad \text { for large } n .
$$

Hence, by (3.1),

$$
\frac{\sigma_{n}}{n^{3}}=\frac{\mu_{n}}{\mu_{n, n}} \frac{\mu_{n, n-2}-1}{\mu_{n}-1} \sim \frac{1}{\mu_{n}\left(\mu_{n}-1\right)}=O\left(\frac{n}{\log n}\right) \quad \text { as } n \rightarrow \infty,
$$

and the second half of (3.7) follows by (3.11).

We can see that (3.8) holds by induction. For, it is immediately satisfied when $n=N_{0}$. When $n=m>N_{0}$ we have, by (3.5) and (3.8) with $n=m-1$,

$$
2 \sum_{k=3}^{m} \alpha_{k}=2 \alpha_{m}+2 \sum_{k=3}^{m-1} \alpha_{k}=m^{3}+2 m^{2}+4 m+2+\tau_{m} .
$$

Finally (3.9) follows from (3.4), (3.6), and (3.8), and (3.10) follows from (3.7).

Next we define the sequence $\left(a_{n}\right)_{n \in \mathbb{N}}$ recursively, and for each $n \in \mathbb{N}$ put

$$
b_{n}=a_{n}-\frac{2}{T_{n}+2} a_{n}=\frac{T_{n}}{T_{n}+2} a_{n} .
$$

Choose $a_{3}$ and $N_{1}$ large, and set $a_{n+1}=a_{n}^{n^{3}}$, for $3 \leq n<N_{1}$. We assume that $a_{3}$ and $N_{1}$ are chosen sufficiently large for various estimates in the sequel to hold. For $n \geq N_{1}$, we define

$$
a_{n+1}=\frac{\left(T_{n+1}+2\right)}{T_{n+1}} b_{n}\left(1-\frac{b_{n}}{a_{n}}\right)^{2} \prod_{k=3}^{n-1}\left\{\left(1-\frac{b_{n}}{a_{k}^{\beta_{k}}}\right)^{2 \alpha_{k}} \prod_{l=0}^{k-2}\left(1-\frac{b_{n}}{a_{k}^{\mu_{k, l}}}\right)^{2}\right\}
$$


Finally in this section we prove a set of inequalities which concern the growth of the sequence $\left(a_{n}\right)$, and the ratios of these numbers to the modulus of the other zeros of $f$. Note that (3.7) and (3.17) imply that the product in (2.1) is locally uniformly convergent in $\mathbb{C}$.

Lemma 3.2. The following inequalities hold for the sequence $\left(a_{n}\right)$ defined above. For $n \geq 3$,

$$
\begin{aligned}
& a_{n}^{n^{3}-2 / n} \leq a_{n+1} \leq a_{n}^{n^{3}+2 / n}, \\
& a_{n}>\exp \left(e^{n}\right),
\end{aligned}
$$

and, for large $n$,

$$
\begin{array}{rlrl}
\frac{a_{n}}{a_{n}^{\mu_{n}}} \leq \exp \left(-e^{n / 2}\right), & \frac{\alpha_{n} a_{n}}{a_{n}^{\beta_{n}}} \leq \exp \left(-e^{n / 2}\right), \\
\frac{a_{n}^{\mu_{n, n-2}}}{a_{n+1}} \leq \exp \left(-e^{n / 2}\right), & \frac{\alpha_{n} a_{n}^{\beta_{n}}}{a_{n+1}} & \leq \exp \left(-e^{n / 2}\right) .
\end{array}
$$

Proof. First, assume that (3.16) holds for $3 \leq n \leq m$. Equation (3.17) follows for $3 \leq n \leq m$ by a simple induction. Hence, for sufficiently large $m$, by (3.7), (3.13), (3.16) and (3.17):

$$
\begin{aligned}
& \frac{a_{m}}{a_{m}^{\mu_{m}}}=a_{m}^{1-\mu_{m}} \leq \exp \left(e^{m}\left(1-\mu_{m}\right)\right) \leq \exp \left(-e^{m / 2}\right) ; \\
& \frac{\alpha_{m} a_{m}}{a_{m}^{\beta_{m}}} \leq 3 m^{2} a_{m}^{1-m^{2} / 2} \leq \exp \left(-e^{m / 2}\right) ; \\
& \frac{a_{m}^{\mu_{m, m-2}}}{a_{m+1}} \leq\left(a_{m}^{\mu_{m, m-2}}\right)^{1-\mu_{m, 2}+\frac{2}{m}} \leq \exp \left(e^{m}\left(1-\mu_{m, 2}+\frac{2}{m}\right)\right) \leq \exp \left(-e^{m / 2}\right) ; \\
& \frac{\alpha_{m} a_{m}^{\beta_{m}}}{a_{m+1}} \leq 3 m^{2} a_{m}^{m^{2}-m^{3}+2 / m} \leq \exp \left(-e^{m / 2}\right) .
\end{aligned}
$$

It remains to prove (3.16). We can assume, by taking $N_{1}$ sufficiently large, that (3.16) holds for $3 \leq n \leq m-1$, for some large $m$. We can assume also that $m$ is sufficiently large that (3.9), (3.10) and various other estimates used in the following hold. We need to prove that (3.16) holds for $n=m$. Now, by (3.15) 
and (3.3),

$$
\begin{aligned}
a_{m+1} & =\frac{\left(T_{m+1}+2\right)}{T_{m+1}} b_{m}^{\kappa_{m}}\left(1-\frac{b_{m}}{a_{m}}\right)^{2} \frac{L_{1}}{\prod_{k=3}^{m-1}\left\{a_{k}^{2 \alpha_{k} \beta_{k}} \prod_{l=0}^{k-2} a_{k}^{2 \mu_{k, l} l}\right\}} \\
& =k_{m} \frac{a_{m}^{\kappa_{m}}}{a_{m-1}^{2 \alpha_{m-1} \beta_{m-1}} \prod_{l=1}^{m-3} a_{m-1}^{2 \mu_{m-1}}} \frac{L_{1}}{L_{2}} \\
& =k_{m} \frac{a_{m}^{\kappa_{m}}}{a_{m-1}^{(m-1)^{3} \tau_{m-1}}} \frac{L_{1}}{L_{2}}
\end{aligned}
$$

where, by (3.14),

$$
k_{m}=\frac{\left(T_{m+1}+2\right)}{T_{m+1}}\left(\frac{T_{m}}{T_{m}+2}\right)^{\kappa_{m}}\left(\frac{2}{T_{m}+2}\right)^{2},
$$

by $(3.9)$,

$$
\begin{gathered}
\kappa_{m}=1+2 \sum_{k=3}^{m-1} \alpha_{k}+\sum_{k=3}^{m-1} \sum_{l=0}^{k-2} 2=m^{3}+\tau_{m-1}=T_{m}, \\
L_{1}=\prod_{k=3}^{m-1}\left\{\left(1-\frac{a_{k}^{\beta_{k}}}{b_{m}}\right)^{2 \alpha_{k}} \prod_{l=0}^{k-2}\left(1-\frac{a_{k}^{\mu_{k} l}}{b_{m}}\right)^{2}\right\}
\end{gathered}
$$

and, by (3.3) again,

$$
\begin{aligned}
L_{2} & =a_{m-1}^{2} \prod_{k=3}^{m-2}\left\{a_{k}^{2 \alpha_{k} \beta_{k}} \prod_{l=0}^{k-2} a_{k}^{2 \mu_{k, l}}\right\} \\
& =a_{m-1}^{2} \prod_{k=3}^{m-2} a_{k}^{2\left(1+\alpha_{k} \beta_{k}+\sum_{l=1}^{k-2} \mu_{k, l}\right)} \\
& =a_{m-1}^{2} \prod_{k=3}^{m-2} a_{k}^{2+k^{3} \tau_{k}} .
\end{aligned}
$$

We now estimate the terms in this equality. Firstly, by (3.6), and noting that $\left(T_{m} /\left(T_{m}+2\right)\right)^{T_{m}}>1 / e^{2}$, we obtain

$$
\frac{1}{8} m^{-6}<k_{m}<8 m^{-6} .
$$

Secondly, by (3.16), with $n=m-1$,

$$
a_{m-1}^{(m-1)^{3}} \leq a_{m}^{\left(1-\frac{2}{(m-1)^{4}}\right)^{-1}} \leq a_{m}^{1+\frac{4}{(m-1)^{4}}}
$$


and so, by (3.4),

$$
\frac{a_{m}^{\kappa_{m}}}{a_{m-1}^{(m-1)^{3} \tau_{m-1}}} \geq a_{m}^{\kappa_{m}-\tau_{m-1}-\frac{4 \tau_{m-1}}{(m-1)^{4}}}=a_{m}^{m^{3}-\frac{4 \tau_{m-1}}{(m-1)^{4}}} \geq a_{m}^{m^{3}-\frac{1}{m}} .
$$

Similarly, by (3.4) and (3.16),

$$
\frac{a_{m}^{\kappa_{m}}}{a_{m-1}^{(m-1)^{3} \tau_{m-1}}} \leq a_{m}^{m^{3}+\frac{1}{m}}
$$

Thirdly, we consider $L_{1}$. Noting (3.10), and by (3.14) and (3.19), we see that the smallest term in this product is

$$
1>\left(1-\frac{a_{m-1}^{\mu_{m-1,3-3}}}{b_{m}}\right)^{2}>\left(1-\frac{2 a_{m-1, m-3}^{\mu_{m-1,3}}}{a_{m}}\right)^{2}>1-\exp \left(-e^{\frac{m}{4}}\right) .
$$

There are fewer than $m$ terms in $L_{1}$ of the form $\left(1-a_{m-1}^{p} / b_{m}\right)^{q}, p \in \mathbb{R}, q \in \mathbb{N}$, and so of comparable size to this term. The other terms in the product for $L_{1}$ tend to 1 sufficiently quickly, by (3.16), that $1 / 2<L_{1}<1$.

Finally, we consider $L_{2}$. Observe that, by (3.4) and (3.16), the largest term in this product is

$$
a_{m-1}^{2} a_{m-2}^{2+(m-2)^{3} \tau_{m-2}}<a_{m-1}^{2} a_{m-2}^{4(m-2)^{4}}<a_{m-1}^{2} a_{m-1}^{8(m-1)}<a_{m}^{16 / m^{2}} .
$$

By (3.16), all other terms in the product for $L_{2}$ decrease sufficiently quickly that $1<L_{2}<a_{m}^{32 / m^{2}}$.

Thus, by (3.20), for sufficiently large $m$,

$$
a_{m+1} \geq \frac{1}{16} m^{-6} a_{m}^{m^{3}-\frac{1}{m}-\frac{32}{m^{2}}} \geq a_{m}^{m^{3}-\frac{2}{m}}
$$

and similarly,

$$
a_{m+1} \leq 8 m^{-6} a_{m}^{m^{3}+\frac{1}{m}} \leq a_{m}^{m^{3}+\frac{2}{m}} .
$$

This completes the proof of Lemma 3.2.

\section{There are no multiply Connected Fatou Components}

In this section we prove the following result.

Lemma 4.1. The transcendental entire function $f$ does not have multiply connected Fatou components. 
We use the definition of an annulus

$$
A\left(r_{1}, r_{2}\right)=\left\{z: r_{1}<|z|<r_{2}\right\}, \quad \text { for } 0<r_{1}<r_{2} .
$$

We need the following, which is part of [6, Theorem 1.2].

Theorem A. Suppose that $g$ is a transcendental entire function with a multiply connected Fatou component $U$. For each $z_{0} \in U$ there exists $\alpha>0$ such that, for sufficiently large $n \in \mathbb{N}$,

$$
g^{n}(U) \supset A\left(\left|g^{n}\left(z_{0}\right)\right|^{1-\alpha},\left|g^{n}\left(z_{0}\right)\right|^{1+\alpha}\right) .
$$

Proof of Lemma 4.1. Observe that, for large $n$, in the closed annulus $\overline{A\left(a_{n}, a_{n+1}\right)}$ there are zeros of $f$ on the negative real axis of modulus $a_{n}, a_{n}^{\mu_{n}}, a_{n}^{\mu_{n, 2}}, \ldots, a_{n}^{\mu_{n, n-2}}$ and $a_{n+1}$. Note also that 0 is a fixed point of $f$, and so no zero of $f$ can be in a multiply connected Fatou component of $f$. Now, by (3.16),

$$
a_{n+1} \leq a_{n}^{n^{3}+2 / n}<\left(a_{n}^{\mu_{n, n-2}}\right)^{\mu_{n, 2}+2 / n} .
$$

Hence, for large $n$, there is at least one zero of $f$ in any annulus $A\left(r, r^{\mu_{n, 2}+2 / n}\right)$, for $a_{n} \leq r \leq a_{n+1}$. Note that $\mu_{n, 2}+2 / n \rightarrow 1$ as $n \rightarrow \infty$.

Now, by Theorem A, if $f$ has a multiply connected Fatou component, then there is a $c>1$, and a sequence $\left(r_{i}\right)_{i \in \mathbb{N}}$, tending to infinity, such that the annuli $A\left(r_{i}, r_{i}^{c}\right)$ are contained in multiply connected Fatou components of $f$. This is in contradiction to the observations above regarding the distribution of zeros of $f$ and the fact that these zeros do not lie in multiply connected Fatou components. Hence there can be no multiply connected Fatou components of $f$.

\section{There are Simply COnNected Fatou Components}

Next we show that $f$ has simply connected Fatou components.

Lemma 5.1. Define $B_{n}=\left\{z:\left|z+b_{n}\right|<\delta_{n} b_{n}\right\}$, where $\delta_{n}=n^{-15}$. Then, for large $n$, we have $f\left(B_{n}\right) \subset B_{n+1}$, and $B_{n}$ is contained in a simply connected Fatou component of $f$.

Proof. Suppose that $z \in B_{n}$, in which case $z=-b_{n}+w b_{n}$ where $|w|<\delta_{n}$. We assume throughout this section that $n$ is sufficiently large for various estimates 
to hold. By (2.1), (3.14) and (3.15),

$$
\frac{f(z)}{-b_{n+1}}=I_{1} I_{2}
$$

where

$$
I_{1}=(1-w)\left(1+w \frac{b_{n}}{a_{n}-b_{n}}\right)^{2} \prod_{k=3}^{n-1}\left\{\left(1+w \frac{b_{n}}{a_{k}^{\beta_{k}}-b_{n}}\right)^{2 \alpha_{k}} \prod_{l=0}^{k-2}\left(1+w \frac{b_{n}}{a_{k}^{\mu_{k} l}-b_{n}}\right)^{2}\right\}
$$

and

$$
I_{2}=\prod_{l=1}^{n-2}\left(1+\frac{z}{a_{n}^{\mu_{n, l}}}\right)^{2} \prod_{k=n}^{\infty}\left(1+\frac{z}{a_{k}^{\beta_{k}}}\right)^{2 \alpha_{k}} \prod_{k=n+1}^{\infty} \prod_{l=0}^{k-2}\left(1+\frac{z}{a_{k}^{\mu_{k, l}}}\right)^{2} .
$$

First consider $I_{1}$. This is a polynomial in $w$ of degree $T_{n}+2$, by (3.9). Write

$$
I_{1}=1+\sum_{j=1}^{T_{n}+2} \eta_{j} w^{j}, \quad \text { for } \eta_{j} \in \mathbb{C} .
$$

Then

$$
\left|I_{1}-1\right| \leq\left|\eta_{1} w\right|+\left|\eta_{2} w^{2}\right|+\cdots+\left|\eta_{T_{n}+2} w^{T_{n}+2}\right| .
$$

We consider $\eta_{1}$. We have, by (3.2), (3.9), (3.14), (3.16), (3.17) and (3.19),

$$
\begin{aligned}
\left|\eta_{1}\right| & =\left|1+2 \frac{b_{n}}{b_{n}-a_{n}}+2 \sum_{k=3}^{n-1}\left\{\alpha_{k} \frac{b_{n}}{b_{n}-a_{k}^{\beta_{k}}}+\sum_{l=0}^{k-2} \frac{b_{n}}{b_{n}-a_{k}^{\mu_{k, l}}}\right\}\right| \\
& =\left|1-T_{n}+2 \sum_{k=3}^{n-1}\left\{\alpha_{k}\left(1+\frac{a_{k}^{\beta_{k}}}{b_{n}-a_{k}^{\beta_{k}}}\right)+\sum_{l=0}^{k-2}\left(1+\frac{a_{k}^{\mu_{k, l}}}{b_{n}-a_{k}^{\mu_{k, l}}}\right)\right\}\right| \\
& =2\left|\sum_{k=3}^{n-1}\left\{\alpha_{k} \frac{a_{k}^{\beta_{k}}}{b_{n}-a_{k}^{\beta_{k}}}+\sum_{l=0}^{k-2} \frac{a_{k}^{\mu_{k, l}}}{b_{n}-a_{k}^{\mu_{k, l}}}\right\}\right| \\
& \leq 4\left|n \alpha_{n-1} \frac{a_{n-1}^{\beta_{n-1}}}{a_{n}}+n^{2} \frac{a_{n-1}^{\mu_{n-1, n-3}}}{a_{n}}\right| \\
& \leq \exp \left(-e^{n / 4}\right) .
\end{aligned}
$$

Note that the cancellation in (5.3) occurs because, due to the choice of $b_{n}$ and $T_{n},-b_{n}$ is very close to a critical point of $f$.

Next consider $\eta_{k}$, for $k>1$. Note that the coefficients of $w$ in the product for $I_{1}$ have modulus at most $b_{n} /\left(a_{n}-b_{n}\right)=T_{n} / 2<n^{3}$. Moreover, the degree of $I_{1}$ 
is less than $2 n^{3}$, and so the expansion of (5.1) contains less than $\left(2 n^{3}\right)^{k}$ terms in $w^{k}$. Hence

$$
\left|\eta_{k}\right|<\left(n^{3}\right)^{k} \cdot\left(2 n^{3}\right)^{k}<n^{7 k}, \quad \text { for large } n .
$$

Hence $\left|\eta_{2} w^{2}\right|<n^{-16}$. The other terms in (5.2) decrease sufficiently quickly that

$$
\left|I_{1}-1\right|<2 n^{-16}, \quad \text { for large } n .
$$

Now we consider $I_{2}$. Observe that each term in the product for $I_{2}$ has modulus less than 1 . Hence, since $-\log (1-x) \leq \log (1+2 x)$, for $0<x<1 / 2$, we have, by (3.12), (3.16), (3.17) and (3.18),

$$
\begin{aligned}
0 & <-\log \left|I_{2}\right| \\
& \leq 2\left(\sum_{l=1}^{n-2} \log \left(1+\frac{2|z|}{a_{n}^{\mu_{n, l}}}\right)+\sum_{k=n}^{\infty} \alpha_{k} \log \left(1+\frac{2|z|}{a_{k}^{\beta_{k}}}\right)+\sum_{k=n+1}^{\infty} \sum_{l=0}^{k-2} \log \left(1+\frac{2|z|}{a_{k}^{\mu_{k, l}}}\right)\right) \\
& \leq 8\left(\sum_{l=1}^{n-2} \frac{a_{n}}{a_{n}^{\mu_{n, l}}}+\sum_{k=n}^{\infty} \alpha_{k} \frac{a_{n}}{a_{k}^{\beta_{k}}}+\sum_{k=n+1}^{\infty} \sum_{l=0}^{k-2} \frac{a_{n}}{a_{k}^{\mu_{k, l}}}\right) \\
& \leq 16\left(\exp \left(-e^{n / 2}\right)+\exp \left(-e^{n / 2}\right)+\exp \left(-e^{n / 2}\right)\right) \\
& \leq \exp \left(-e^{n / 4}\right) .
\end{aligned}
$$

Thus, $1-2 \exp \left(-e^{n / 4}\right) \leq\left|I_{2}\right| \leq 1$. This, together with (5.4), establishes the first part of the lemma. It follows from Montel's theorem that, for large $n, B_{n}$ is contained in a Fatou component, which must be simply connected by Lemma 4.1.

Remark. Let $V_{n}$ be the Fatou component containing $B_{n}$. These Fatou components are distinct. For, suppose that $V_{m}=V_{n}$ with $m \neq n$. Because all the coefficients of $z$ in (2.1) are real, the Fatou set $F(f)$ must be invariant under reflection in the real axis. Hence, all points on the negative real axis between $B_{n}$ and $B_{m}$ must be in $V_{m}$, as otherwise $V_{m}$ would be multiply connected. This is a contradiction since these points include the zeros of $f$.

\section{The Simply CONNECted FAtou COMPonents ARE FAST ESCAPING}

In this section we first prove Theorem 2, and then we use this result to prove the following. 
Lemma 6.1. Let $V_{n}, n \in \mathbb{N}$, be the simply connected Fatou components defined at the end of Section 5. Then $V_{n} \subset A(f)$, for large $n$.

Proof of Theorem 2. We need two facts about the maximum modulus function, $M(\rho)$, of a transcendental entire function. The first is well known, and the second is from [9, Lemma 2.2]:

$$
\frac{\log M(\rho)}{\log \rho} \rightarrow \infty \text { as } \rho \rightarrow \infty
$$

and there exists $R>0$ such that

$$
M\left(\rho^{c}\right) \geq M(\rho)^{c}, \quad \text { for } \rho \geq R, c>1 .
$$

Fix $r_{0} \geq R_{0}$ such that $M(r)>r$, for $r \geq r_{0}$. Whenever $r \geq r_{0}$ there is a unique $n \in \mathbb{N}$ such that $M^{n-1}\left(r_{0}\right) \leq r<M^{n}\left(r_{0}\right)$. Hence, since $\epsilon$ is nonincreasing, by (1.1) and (6.1)

$$
\epsilon(r) r \geq \epsilon\left(M^{n}\left(r_{0}\right)\right) M^{n-1}\left(r_{0}\right) \geq \epsilon\left(r_{0}\right)^{n+1} M^{n-1}\left(r_{0}\right) \rightarrow \infty \text { as } n \rightarrow \infty .
$$

Hence

$$
\epsilon(r) r \rightarrow \infty \text { as } r \rightarrow \infty .
$$

By (6.1) and (6.3) we see that, given $k>0$, we can ensure that

$$
\frac{\log M(\epsilon(r) R)}{\log (\epsilon(r) R)}>k, \quad \text { for large } r, R \geq r .
$$

A little algebra shows that this is equivalent to

$$
M(\epsilon(r) R)^{\frac{-\log \epsilon(r)}{\log (\epsilon(r) R)}}>\epsilon(r)^{-k}, \quad \text { for large } r, R \geq r .
$$

In (6.2) we replace $c$ with $\log R / \log (\epsilon(r) R)$, and replace $\rho$ with $\epsilon(r) R$. We obtain, using (6.5) with $k=3$, that there exists $R_{1} \geq R_{0}$ such that

$$
M(R) \geq \epsilon(r)^{-3} M(\epsilon(r) R), \quad \text { for } R \geq r \geq R_{1} .
$$

Note that we can assume that $R_{1}$ is sufficiently large that both $M(r)>r$, for $r \geq R_{1} / \epsilon(r)$, and also, by (6.3), that $\eta(r)>r$, for $r \geq R_{1}$.

We claim next that we have

$$
\eta^{k}(r) \geq \epsilon(r)^{-k-1} M^{k}(\epsilon(r) r)>M^{k}(\epsilon(r) r), \quad \text { for } r \geq R_{1}, k \in \mathbb{N} .
$$


This can be seen by induction. When $k=1$ we have, by (6.6),

$$
\eta(r)=\epsilon(r) M(r) \geq \epsilon(r)^{-2} M(\epsilon(r) r), \quad \text { for } r \geq R_{1} .
$$

Hence, by induction, for $r \geq R_{1}$,

$$
\begin{aligned}
& \eta^{k+1}(r)=\epsilon\left(\eta^{k}(r)\right) M\left(\eta^{k}(r)\right) \\
& \geq \epsilon\left(M^{k}(r)\right) M\left(\eta^{k}(r)\right) \quad \text { as } \epsilon \text { is nonincreasing } \\
& \geq \epsilon(r)^{k+1} M\left(\eta^{k}(r)\right) \\
& \geq \epsilon(r)^{k+1} M\left(\epsilon(r)^{-k-1} M^{k}(\epsilon(r) r)\right) \\
& \geq \epsilon(r)^{k+1} \epsilon(r)^{-3 k-3} M^{k+1}(\epsilon(r) r) \quad \text { by repeated use of (6.6) } \\
& \geq \epsilon(r)^{-(k+1)-1} M^{k+1}(\epsilon(r) r) \quad \text { as required. }
\end{aligned}
$$

(Note that in the penultimate step we have used the fact that $\epsilon(r) r \leq M(\epsilon(r) r)$, for $r \geq R_{1}$.)

It follows from (6.7) that, for $r \geq R_{1}, \eta^{n}(r) \rightarrow \infty$ as $n \rightarrow \infty$. Define

$A^{\prime}(f)=\left\{z:\right.$ there exists $\ell \in \mathbb{N}$ such that $\left|f^{n+\ell}(z)\right| \geq \eta^{n}\left(R^{\prime}\right)$ for $\left.n \in \mathbb{N}\right\}$,

for $R^{\prime} \geq R_{1}$. We complete the proof by showing that $A^{\prime}(f)=A(f)$.

First, suppose that $z \in A(f)$, in which case for some $\ell \in \mathbb{N}$ we have

$$
\left|f^{n+\ell}(z)\right| \geq M^{n}(R), \quad \text { for } n \in \mathbb{N},
$$

and some $R$ with $M(r)>r$, for $r \geq R$.

Choose $K \in \mathbb{N}$ such that $M^{K}(R)=R^{\prime} \geq R_{1}$. Then

$$
\left|f^{n+\ell+K}(z)\right| \geq M^{n+K}(R)=M^{n}\left(R^{\prime}\right) \geq \eta^{n}\left(R^{\prime}\right), \quad \text { for } n \in \mathbb{N} .
$$

Hence $z \in A^{\prime}(f)$.

Conversely, suppose that $z \in A^{\prime}(f)$, in which case for some $\ell \in \mathbb{N}$ and $R^{\prime} \geq R_{1}$ we have

$$
\left|f^{n+\ell}(z)\right| \geq \eta^{n}\left(R^{\prime}\right), \quad \text { for } n \in \mathbb{N} .
$$

Choose $K \in \mathbb{N}$ so that $M^{K}\left(\epsilon\left(R^{\prime}\right) R^{\prime}\right)=R \geq R_{1}$. Then, by (6.7).

$$
\left|f^{n+\ell+K}(z)\right| \geq \eta^{n+K}\left(R^{\prime}\right) \geq M^{n+K}\left(\epsilon\left(R^{\prime}\right) R^{\prime}\right) \geq M^{n}(R), \quad \text { for } n \in \mathbb{N} .
$$

Hence $z \in A(f)$.

Finally, we give the 
Proof of Lemma 6.1. For some large $R_{0}$ define, for $r>R_{0}$,

$$
\epsilon(r)=\frac{1}{16 n^{6}}, \quad \text { for } a_{n-1}\left(1+\delta_{n-1}\right)<r \leq a_{n}\left(1+\delta_{n}\right),
$$

where $\delta_{n}=n^{-15}$ as in Lemma 5.1. Define also $\eta(r)=\epsilon(r) M(r)$, for $r \geq R_{0}$.

Suppose that $x^{\prime} \in B_{n} \cap \mathbb{R} \subset V_{n}$, for some $n$. We can assume that $n$ is chosen sufficiently large for various estimates in this section to hold. We claim that $x^{\prime} \in A(f)$. Thus, by [10, Theorem 1.2], $V_{n} \subset A(f)$.

Our approach to proving this claim is as follows. Set $x=-x^{\prime}$, recalling that $x>0$. We first show that

$$
\left|f\left(x^{\prime}\right)\right| \geq \frac{1}{16 n^{6}} M(x) .
$$

It follows from this, and since $f\left(x^{\prime}\right) \in B_{n+1} \cap \mathbb{R}$, that

$$
\left|f^{m}\left(x^{\prime}\right)\right| \geq \eta^{m}(x), \quad \text { for } m \in \mathbb{N} \text {. }
$$

Second, we show that $\epsilon$ satisfies (1.1). Thus, by (6.10) and Theorem $2, x^{\prime} \in A(f)$, as required.

First we need to establish (6.9). Since $M(x)=f(x)$, we have

$$
\frac{\left|f\left(x^{\prime}\right)\right|}{M(x)}=J_{1} J_{2} J_{3}
$$

where

$$
\begin{aligned}
& J_{1}=\prod_{k=3}^{n-1}\left\{\left(\frac{a_{k}^{\beta_{k}}-x}{a_{k}^{\beta_{k}}+x}\right)^{2 \alpha_{k}} \sum_{l=0}^{k-2}\left(\frac{a_{k}^{\mu_{k, l}}-x}{a_{k}^{\mu_{k, l}}+x}\right)^{2}\right\}, \\
& J_{2}=\left(\frac{a_{n}-x}{a_{n}+x}\right)^{2},
\end{aligned}
$$

and

$$
J_{3}=\prod_{k=n+1}^{\infty}\left\{\left(\frac{a_{k}^{\beta_{k}}-x}{a_{k}^{\beta_{k}}+x}\right)^{2 \alpha_{k}} \sum_{l=0}^{k-2}\left(\frac{a_{k}^{\mu_{k, l}}-x}{a_{k}^{\mu_{k, l}}+x}\right)^{2}\right\}\left(\frac{a_{n}^{\beta_{n}}-x}{a_{n}^{\beta_{n}}+x}\right)^{2 \alpha_{n}} \sum_{l=1}^{n-2}\left(\frac{a_{n}^{\mu_{n, l}}-x}{a_{n}^{\mu_{n, l}}+x}\right)^{2} .
$$


We consider these three terms separately. By (3.10) and (3.19), the smallest term in $J_{1}$ is

$$
\left(\frac{a_{n-1}^{\mu_{n-1, n-3}}-x}{a_{n-1}^{\mu_{n-1, n-3}}+x}\right)^{2}=\left(\frac{1-\frac{a_{n-1}^{\mu_{n-1, n-3}}}{x}}{1+\frac{a_{n-1}^{\mu_{n-1, n-3}}}{x}}\right)^{2} \geq\left(1-\frac{8 a_{n-1}^{\mu_{n-1, n-3}}}{a_{n}}\right) \geq\left(1-\exp \left(-e^{n / 4}\right)\right) .
$$

Thus, recalling the estimates of Lemma $3.2, J_{1} \geq \frac{1}{2}$.

Secondly, recalling that $x=b_{n}+\omega b_{n}$, with $|\omega|<\delta_{n}=n^{-15}$, we have for large $n$,

$$
J_{2}=\left(\frac{a_{n}-b_{n}-\omega b_{n}}{a_{n}+b_{n}+\omega b_{n}}\right)^{2}=\left(\frac{2-\omega T_{n}}{2 T_{n}+2+\omega T_{n}}\right)^{2} \geq \frac{1}{4 n^{6}} .
$$

Thirdly, by (3.10) and (3.18), the smallest term in $J_{3}$ is

$$
\left(\frac{a_{n}^{\mu_{n}}-x}{a_{n}^{\mu_{n}}+x}\right)^{2} \geq\left(\frac{1-\frac{x}{a_{n}^{\mu_{n}}}}{1+\frac{x}{a_{n}^{\mu_{n}}}}\right)^{2} \geq\left(1-\frac{8 a_{n}}{a_{n}^{\mu_{n}}}\right) \geq\left(1-\exp \left(-e^{n / 4}\right)\right) .
$$

Thus, recalling again the estimates of Lemma $3.2, J_{3} \geq \frac{1}{2}$. This establishes our first claim.

Finally, we need to show that $\epsilon$ satisfies (1.1). We claim that $M\left(a_{n}\left(1+\delta_{n}\right)\right) \leq$ $a_{n+2}$. In a very similar way to the proof of $(6.9)$, we can split $f\left(2 b_{n}\right) / a_{n+1}$ into three terms, and show that $f\left(2 b_{n}\right) / a_{n+1}<2^{12 n^{3}}$. Hence, by (3.14), (3.16) and (3.17),

$$
M\left(a_{n}\left(1+\delta_{n}\right)\right) \leq M\left(2 b_{n}\right)=f\left(2 b_{n}\right)<2^{12 n^{3}} a_{n+1} \leq a_{n+1}^{2} \leq a_{n+2},
$$

as required.

Suppose then that $r$ is such that $a_{n-1}\left(1+\delta_{n-1}\right)<r \leq a_{n}\left(1+\delta_{n}\right)$. Since $\epsilon$ is nonincreasing, we deduce that, for $k \in \mathbb{N}$,

$$
\begin{aligned}
\epsilon\left(M^{k}(r)\right) & \geq \epsilon\left(M^{k}\left(a_{n}\left(1+\delta_{n}\right)\right)\right) \\
& \geq \epsilon\left(a_{n+2 k}\right) \\
& =\frac{1}{16(n+2 k)^{6}} \geq \frac{1}{\left(16 n^{6}\right)^{k+1}}=\epsilon(r)^{k+1} .
\end{aligned}
$$

Thus $\epsilon$ satisfies (1.1). This completes the proof of Lemma 6.1 and hence the proof of Theorem 1. 
Acknowledgement. The author is grateful to Gwyneth Stallard and Phil Rippon for their help with this paper.

\section{REFERENCES}

[1] BAKER, I. N. Multiply connected domains of normality in iteration theory. Math. Z. 81 (1963), 206-214.

[2] BAKER, I. N. Some entire functions with multiply-connected wandering domains. Ergodic Theory Dynam. Systems 5, 2 (1985), 163-169.

[3] Bergweiler, W. Iteration of meromorphic functions. Bull. Amer. Math. Soc. (N.S.) 29, 2 (1993), 151-188.

[4] Bergweiler, W. An entire function with simply and multiply connected wandering domains. Pure Appl. Math. Quarterly 7, 2 (2011), 107-120.

[5] Bergweiler, W., And Hinkkanen, A. On semiconjugation of entire functions. Math. Proc. Cambridge Philos. Soc. 126, 3 (1999), 565-574.

[6] Bergweiler, W., Rippon, P. J., and Stallard, G. M. Multiply connected wandering domains of entire functions. Preprint, arXiv:1109.1794v1 (2011).

[7] Kisaka, M., AND Shishikura, M. On multiply connected wandering domains of entire functions. In Transcendental dynamics and complex analysis, vol. 348 of London Math. Soc. Lecture Note Ser. Cambridge Univ. Press, Cambridge, 2008, pp. 217-250.

[8] Rippon, P. J., And Stallard, G. M. On questions of Fatou and Eremenko. Proc. Amer. Math. Soc. 133, 4 (2005), 1119-1126.

[9] Rippon, P. J., And Stallard, G. M. Functions of small growth with no unbounded Fatou components. J. Anal. Math. 108 (2009), 61-86.

[10] Rippon, P. J., And Stallard, G. M. Fast escaping points of entire functions. To appear in Proc. London Math. Soc., arXiv:1009.5081v1 (2011).

\section{J. Sixsmith}

Department of Mathematics and Statistics

The Open University

Walton Hall

Milton Keynes MK7 6AA

UK

E-mail: david.sixsmith@open.ac.uk 